for such polycrystalline materials and mentioned the increasing importance of automatic counter recording. Powder diagrams were shown to illustrate the identification of metals, alloys and slags with regard to chemical nature (fingerprinting), physical texture, and particle size.

The last session dealt with diffraction of radiation other than X-rays. Dr. H. Wilman (Imperial College of Science and Technology) described the-sometimes rather ad hoc-apparatus used for observing the very intense scattering of electrons and illustrated the range of patterns which may arise. After mentioning the fairly complete structural information which has been obtained from some rubbers and from single crystals, Dr. Wilman discussed recent electron diffraction studies carried out at the Imperial College of Science and Technology on reactions at surfaces. Much of the discussion which followed was concerned with deriving information of use to metallurgists about surface structure.
In the final talk, on neutron diffraction applications, Mr. N. A. Curry (Atomic Energy Research Establishment, Harwell) pointed out that typically a neutron beam would have an effective intensity less than onethousandth of that from an X-ray diffraction source, and, moreover, it covered a range of wave-lengths, so that at most reactors both apparatus and specimen had to be large by standards normally used with $\mathrm{X}$-ray techniques. The markedly different scattering power of nuclei to neutrons (only elastic scattering was considered in any detail), as compared with the corresponding $\mathrm{X}$-ray atomic scattering factors, was the basis of the chief merit of neutron diffraction. In particular (and the subsequent lively discussion centred on this), hydrogen atoms had been located with a precision of $0.03 \AA$. in compounds containing atoms of appreciable atomic number, for example, in the study of the symmetry of short $\mathrm{O}-\mathrm{H}$. . . O bonds in potassium hydrogen bis-phenylacetate.

D. W. JoNES

\title{
PLANT TISSUE AND ORGAN CULTURE
}

A SYMPOSIUM on "Plant Tissue and Organ Culture" was held in the Department of Botany, University of Delhi, during December 22-29, under the auspices of the Unesco South Asia Science Cooperation Office, New Delhi, and the University of Delhi. Prof. P. Maheshwari was the convener. Fifty delegates from Burma, Ceylon, France, West Germany, India, Malaya, the United Kingdom and the United States participated.

The 36 papers presented covered a wide range of subjects, and they show the diversity of purposes for which the technique of plant tissue culture can be used. Quite a few of the papers were concerned with the broad subject of physiology of reproductive processes in angiosperms and involved the culturing of flowers, ovaries, ovules, anthers, etc.

The tissue culture technique has contributed in recent years to a better understanding of a variety of morphogenetic problems and naturally this aspect found a fair representation at the symposium. The culturing of parts of parasitic higher plants is of special interest from the physiological point of view and there were three papers on this subject.

Embryos are among the material most often used for in vitro culture for a long time, and interest in them still continues as ean be seen from the three papers dealing with this means of approach.

Some investigators are interested in tissue culture techniques as an aid in propagation of horticultural plants as seen in two papers presented. Isolated tissues provide a valuable means for the bioassay of growth substances and cell division factors, and several authors described their investigations along these lines.

Studies of abnormal growth might sometimes lead to an elucidation of normal growth processes besides throwing light on the nature of carcinogenic growth in general. From this point of view one paper was of interest.

Knowledge of the nutrition of tissue cultures is of basic importance for the successful growth of tissue cultures of all types, especially since many tissues seem to have certain specific nutritional requirements. This was indicated in three papers. Other aspects of tissue culture methods were dealt with by still further authors.

In addition to the special papers read, a few special lectures were also arranged. Prof. F. C. Steward (Cornell), in his lecture entitled "Carrots and Coconuts: Some Investigations on Growth", dealt at length with the importance of coconut milk and other growth factors with special reference to his own recent work on the culturing of cell suspensions of carrot and of Haplopappus gracilis, the angiosperm with the lowest number of chromosomes $(2 n=4)$. Prof. H. E. Street (Swansea) gave two lectures, both concerned with root growth. In the first he summarized the work done by himself and his coworkers on the role of various sugars, nitrogen sources and other nutritional factors in root cultures. The second gave an exposition of the rather tricky question of the part played by auxins in root growth, tracing the history of the subject with special emphasis on the work done in Prof. Street's labora. tory. Prof. J. Reinert (Berlin-Dahlem) described the formation of adventive embryos and pro-embryolike structures in carrot cultures grown on a purely synthetic medium containing vitamins, amino-acids and hypoxanthine. The differentiation of shoots could be prevented by addition of coconut milk to the medium. Prof. J. P. Nitsch (Gif-sur-Yvette), who was one of the earliest to culture successfully isolated ovaries and flowers of higher plants in vitro, gave an account of some of his recent experiments at the phytotron, France, on the in vitro culture of flowers and fruits of tomato and other plants. Prof. P. Maheshwari (Delhi) spoke on "Plant Tissue and Organ Culture from an Embryologist's Point of View"

The symposium concluded with a discussion on "Techniques: Appraisal of Progress and Future Outlook on Tissue Culture Research" in which several participants expressed the view that in future there should be more emphasis on the biochemical fate of the various growth substances added to the medium and the cytological phenomena accompanying growth of tissue cultures.

R. L. N. SASTRI 